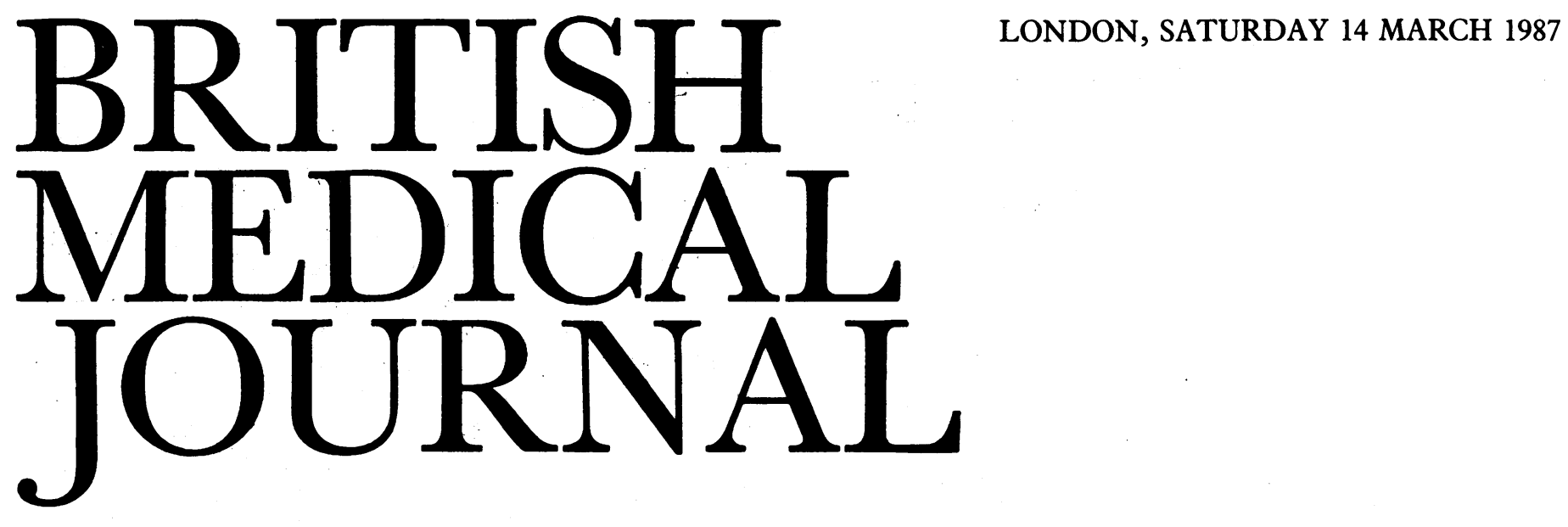

\title{
Do adhesions cause pain?
}

Peritoneal damage from infection, haemorrhage, radiation, and particularly operative trauma may result in adhesions of one peritoneal surface to the other. These adhesions may vary from a diaphanous strand to an encircling tough cocoon. Their formation is probably important for defence, and the omentum or "abdominal policeman". is the most prolific former of abdominal adhesions. Lesser members of the intraabdominal defence force-such as the epiploic appendicescan mimic their master. Adhesions allow the omentum to seal off inflammation and limit the damage or contain infection. A specimen in the anatomical museum in Vienna shows a human stomach perforated by a spoon that has become wrapped by omental adhesions and isolated from the rest of the peritoneal cavity. ${ }^{1}$

Though adhesions may have good intentions, they also have drawbacks-as every abdominal surgeon knows. They induce surgical tedium, and abdominal reoperations can be time consuming and sometimes dangerous. Adhesions can turn what is expected to be a simple laparotomy into a long ordeal of dividing adhesions. Furthermore, adhesions behind a previous laparotomy wound provide dangers for the unwary surgeon, who may, embarrassingly, incise his way into the lumen of the gut in mistake for the peritoneal cavity. Surgeons and adhesions are thus often not the best of friends. An adhesion may also act as a snare for the unwary viscus. It is common to find acute intestinal obstruction caused by a long fibrous adhesion lassooing and sometimes strangling a loop of gut. Though all abdominal surgeons accept that adhesions cause laparotomy hazards, operative tedium, and strangulation, controversy surrounds the question whether adhesions produce intra-abdominal or pelvic pain.

I believe it to be a poorly substantiated myth that adhesions can cause abdominal or pelvic pain. ${ }^{1.4}$ The blame for perpetuating the myth rests partly with surgeons who are driven to perform diagnostic laparotomies on patients with functional disorders. They may find a few adhesions and then announce to the patient that the cause of the pain has been discovered and that once the adhesions are divided all will be well. The blame also rests partly with the neurotic patients themselves, who are desperate for an explanation for their symptoms that will protect them from the feared label of "neurotic." Some patients' symptoms will resolve com- pletely after the laparotomy and explanation, and even more will have the regulation postoperative "honeymoon" that characterises many placebo operations. Then their symptoms may return and be blamed on new adhesion formationwell, what do you expect?

But can adhesions cause symptoms? Are they worth dividing, and should steps be taken to prevent the formation of further adhesions? No clear answers are available; only opinions. A scientific trial to determine whether adhesions can cause symptoms is difficult to plan because randomised controls are hard to find. Although there are many anecdotes of patients being relieved of specific abdominal symptoms by adhesions being divided, a placebo effect cannot be excluded.

A recent study from Los Angeles reports a retrospective analysis of 100 consecutive diagnostic laparoscopies performed for chronic pelvic pain. ${ }^{5}$ These results were compared with those at 88 consecutive laparoscopies for infertility in women of whom only four had important pelvic pain. A quarter of the patients who were investigated for pain had pelvic adhesions compared with two fifths in the infertility group. The author concluded that the results seriously question whether pelvic adhesions cause pain. He investigated the possibility that the site and nature of the adhesions were different in the group having investigations for infertility, but there was no significant difference. Performing a similar study to determine whether abdominal as opposed to pelvic adhesions ever cause symptoms would be difficult as there is no justification for performing routine laparoscopy in the abdomen.

Some surgeons claim anecdotal "xperience of patients with sharp localised abdominal pain behind a previous scar. Laparoscopic examination showed that they had band like adhesions, and division of them brought immediate relief. All who, like me, relate such experiences should beware of the powerful placebo effect of the procedure. Without scientifically reliable data or the prospect of clinical trials abdominal surgeons should be cautious about arguing that adhesions cause abdominal pain. They should also beware of the danger of skewering the gut during laparoscopy in a patient likely to have intra-abdominal adhesions.

Sometimes all abdominal adhesions must be divided when reoperating to sort out previous surgical failures. Such 
division is performed to allow total gut assessment, not in the hope of curing or preventing symptoms. A single tough band like adhesion should, however, be divided to prevent possible strangulation. In my opinion there is no scientifically reliable evidence in man that washing out the peritoneum or instilling antiseptics, anti-inflammatory drugs, or antibiotics reduces adhesion formation.

JOHN ALEXANDER-WILLIAMS

Consultant Surgeon,

General Hospital,

Birmingham B4 6NH

1 Robert HLF. De statu morboso omenti. Marburg, 1840:32, 14-6, 23-4.

2 Goldstein DP, deCholnoky C, Emans SJ, Leventhal JM. Laparoscopy in the diagnosis and management of pelvic pain in adolescents. $\mathcal{F}$ Reprod Med 1980;24:251-6.

$3 \mathrm{Kresh}$ AJ, Seifer DB, Sachs LB, Barrese I. Laparoscopy in 100 women with chronic pelvic pain Obstet Gynecol 1984;64:672-4.

Malinak LR. Operative management of pelvic pain. Clin Obstet Gynecol 1980;23:191-200.

5 Rapkin AJ. Adhesions and pelvic pain: a retrospective study. Obstet Gynaecol 1986;68:13-5.

\section{Wellcome developments in tropical medicine}

The seemingly conflicting aims of Sir Henry Wellcome to provide educational entertainment for the casual visitor and serious research material for the student continue to be fulfilled by the Wellcome Tropical Institute. Established by the Wellcome Trust in 1984 the institute is working, firstly, to update and develop the Wellcome Museum of Medical Science and, secondly, to help governments and universities in the tropics to run their own courses in tropical medicine and to develop continuing education for medical officers away from teaching hospitals.

The two ventures are closely intertwined. As well as revising existing museum material and acquiring fresh exhibits the institute hopes to hold special exhibitions every two years on topics of current interest in tropical medicine. The first, on malaria, opens next week and is both visually striking and informative (p 709). It has clear, detailed diagrams and micrographs of the stages in the life cycle of both the anopheles mosquito and the plasmodium parasite and a broader overview of the problems of control and treatment. As well as inviting sixth formers and undergraduates to visit the exhibition the institute is sending copies of the exhibition panels to medical schools in Africa. Schools in Nairobi and Addis Ababa have already received material.

More ambitious is the institute's aim to develop a distance learning programme for continuing postgraduate education. The programme, devised for district medical officers in rural areas, is being worked out in collaboration with governments and universities to ensure that the methods of self instruction, including slide-sound and video, are suitable. As Dr Alan Knell, deputy director of the institute, points out, sending display materials over is easy compared with implementing the programme at the other end. More input and organisation are needed. Nevertheless, plans are progressing, and Dr Knell hopes that programmes will start later this year in Kenya and Addis Ababa. Sir Henry, I feel, would approve.

KIM WINTER

Subeditor,

$B M \mathcal{F}$

\section{Through the carpal tunnel}

The carpal tunnel syndrome is the commonest entrapment neuropathy, affecting the median nerve as it lies between the carpal bones, the flexor tendons, and the unyielding transverse carpal ligament. A general practitioner may expect to see on average two patients each year with the syndrome. ${ }^{1}$ It affects women between two and 10 times more often then men, is most common between 30 and 50 years of age, and is bilateral in one third of patients. ${ }^{1}$

The carpal tunnel syndrome is secondary to other conditions in $10-50 \%$ of patients, and in general practice it is seen most often with pregnancy, the premenstrual syndrome, and obesity. The tunnel may be narrowed by disruption of its bony architecture, by thickening of the synovium and flexor tendon sheaths, or by tumours and deposits. Thus the syndrome may be secondary to Colles' fracture and other bony deformities (as first described by James Paget ${ }^{2}$ ); to rheumatoid arthritis, and arthritides caused, for instance, by rubella, or to tuberculous tenosynovitis; to lipomas, and to amyloid or tophaceous deposits; and to the increased venous engorgement caused by inserting an arteriovenous shunt for haemodialysis. General conditions that affect the carpal tunnel include myxoedema, acromegaly, diabetes, polycythaemia vera, and haemolytic anaemias.

Diagnosis in general practice depends on an accurate history, examination, and various provocative tests. In hospital these are supplemented by electrodiagnosis. The characteristic symptoms are nocturnal tingling and pain in the thumb, index, and middle fingers of one or both hands; the paraesthesia may spread to other fingers, the hand, and the forearm and may be accompanied by numbness. Often symptoms are precipitated by unaccustomed use of the hands and initially are relieved by shaking the hand or hanging it out of bed. Stiffness or clumsiness of the fingers is experienced by less than half of patients, and few complain of swollen fingers.

Usually nothing abnormal is found on examination. Thenar wasting is present in only one third of patients, and less than one sixth of patients have objective hypoaesthesia of the finger tips. Provocation tests are thus an essential adjunct to diagnosis. In the Phalen test symptoms are produced or exacerbated by complete flexion of the wrist for 30-60 seconds. ${ }^{3}$ A positive Tinel's test demands producing tingling in the fingers by gently tapping the area over the median nerve in the wrist. In the tourniquet test a blood pressure cuff applied to the upper arm is inflated above systolic blood pressure for 60 seconds; the production or exacerbation of symptoms in the fingers means the test is positive. Nerve conduction studies may show reduced conduction velocities in the median nerve as it crosses the wrist, with changes in sensory nerves preceding those in motor nerves; distal motor latency time may be prolonged. Alternatively, results may be within normal limits but show slower nerve conduction on the affected side.

Gellman and colleagues have recently assessed the sensitivity and specificity of provocation tests in comparison with electrodiagnosis. ${ }^{4}$ Phalen's test was most useful-positive in three quarters of patients and falsely positive in only $20 \%$ of controls. The tourniquet test was the least reliable-only $65 \%$ of patients were positive and $40 \%$ of controls were falsely positive. Tinel's percussion test was interesting because, although relatively insensitive with only $44 \%$ of patients positive, it was highly specific with only $6 \%$ of false 